(C) 2017. This manuscript version is made available under the CC-BY-NC-ND 4.0 license http://creativecommons.org/licenses/by-nc-nd/4.0/

\title{
Searching objective criteria for patient assignment in addiction treatment
}

Running head: Patient assignment criteria in addictions

José J. López-Goñi ${ }^{\mathrm{a}}$, Javier Fernández-Montalvo ${ }^{\mathrm{a}}$, Alfonso Arteaga ${ }^{\mathrm{a}}$, and Sonia Esarte ${ }^{\mathrm{a}}$ a Departamento de Psicología y Pedagogía

Universidad Pública de Navarra

31006 Pamplona

Spain

Correspondence concerning this article should be addressed to José J. López-Goñi,

Dpto. Psicología y Pedagogía, Universidad Pública de Navarra, Campus de Arrosadía s/n, 31006 (Navarra) Spain. E-mail: josejavier.lopez@unavarra.es

Journal of Substance Abuse Treatment, 2017, 76, 28-35

http://dx.doi.org/10.1016/j.jsat.2017.02.014 


\begin{abstract}
The main aim of this study was to objectify the treatment assignment criteria used in a clinical centre for addiction treatment in Spain. A sample of 162 patients (87 inpatients and 75 outpatients) who sought treatment between 2010 and 2012 was assessed. Clinical characteristics (addiction severity, psychopathological symptoms, impulsiveness and maladjustment) of the two treatment groups (inpatient and outpatient) into which patients were assigned according to the clinical criteria of therapists were analysed to identify which variables were more relevant for patient placement. Moreover, the therapeutic progression of patients who met and did not meet the assignment criteria received was studied. According to the results, a score above 4 in the family/social support area of the European Addiction Severity Index (EuropASI), or, in cases of a score between 2 and 4 in the family/social area of EuropASI, a score above 2 in the partner subscale of the Maladjustment Scale correctly classified 73.5\% of cases (96.6\% of inpatients and $46.7 \%$ of outpatients). Comparisons of therapeutic results depending on matching or mismatching these assignment criteria showed a larger effect size in mismatching patient assignment criteria for outpatient treatment. The results obtained in this study provide an objective criterion for addicted patient placement. Moreover, from a cost-effective perspective, they question the necessity of inpatient treatment in most cases, demonstrating that outpatient treatment is a sufficient level of care. This study addresses the approach to assigning patients to the treatment modality that best fits them, implementing the least expensive level of care needed to achieve treatment success.
\end{abstract}

Keywords: Drug addiction; therapeutic assignment criteria; therapeutic community; outpatient treatment; effectiveness.

Journal of Substance Abuse Treatment, 2017, 76, 28-35 
Journal of Substance Abuse Treatment, 2017, 76, 28-35

http://dx.doi.org/10.1016/j.jsat.2017.02.014 


\section{Introduction}

The cost-effectiveness of treatment for alcohol and drug abuse disorders is currently an important challenge in the field of addictions. Adequate treatment matching based on empirically established patient placement criteria becomes relevant to optimally provide cost- and outcome-effective treatment (McGee \& MeeLee, 1997). The correct assignment to an inpatient or outpatient treatment modality based on the needs of patients is critical in optimizing the clinical intervention provided.

The literature about addiction treatment effectiveness explains that both inpatient and outpatient modalities have empirical evidence supporting their effectiveness (Fernández-Montalvo \& López-Goñi, 2010; Fernández-Montalvo, López-Goñi, Illescas, Landa, \& Lorea, 2008; Hubbard, Craddock, \& Anderson, 2003; Magor-Blatch, Bhullar, Thomson, \& Thorsteinsson, 2014; McCarty et al., 2014; Reif et al., 2014; Vanderplassschen et al., 2013; Wallace \& Weeks, 2004). However, research with greater specificity and consistency is needed, taking into account the heterogeneity of both inpatient (e.g. therapeutic community versus short-term residential treatments) and outpatient (e.g. regular versus intensive) treatment programmes.

In clinical settings, the decision about which treatment modality (outpatient or inpatient) is the most appropriate for each patient is often based on the subjective criteria of therapeutic teams. There is an important lack of objective clinical criteria matching patients to the type of treatment best suited to their needs. Therapists use their clinical impression of the patient's situation and tend to assign inpatient treatment to those with more severe addictions as well as those who do not have good family or partner support to help them in the recovery process (Gregoire, 2000; Harrison \& Asche, 1999). Another common criterion used to assign inpatient treatment is the lack of satisfactory results in outpatient programmes. Patients with frequent episodes of Journal of Substance Abuse Treatment, 2017, 76, 28-35 
relapse or high dropout rates in outpatient treatment are usually moved to inpatient programmes (Gregoire, 2000; López-Goñi, Fernández-Montalvo, Cacho, \& Arteaga, 2014).

However, it is necessary to determine objective criteria beyond the clinical impression of therapists that will enable decision making based on empirical evidence when assigning patients to the most appropriate treatment modality. Although there have been some attempts to establish objective patient placement criteria (American Society of Addiction Medicine, 1996; Hoffman, Halikas, Mee-Lee, \& Weedman, 1991; McGee \& MeeLee, 1997; McKay, Cacciola, McLellan, Alterman, \& Wirtz, 1997; Stallvik, Gastfriend, \& Nordahl, 2015), there is still not a scientific consensus on which criteria should be taken into account to place patients in the best level of care. This is an important issue because one of the main challenges in the treatment of addictions focuses on matching treatment to the specific needs of each patient (Camilleri, Cacciola, \& Jenson, 2012; Gregoire, 2000; McGee \& MeeLee, 1997; McKay et al., 1997; Rohrig, Buchholz, Wahl, \& Berner, 2015).

Therefore, the main purposes of this naturalistic study were to objectify the treatment assignment criteria used in a clinical centre for addictions in Spain and to evaluate the subsequent treatment progression. The specific goals were first, to analyse the clinical characteristics (addiction severity, psychopathological symptoms, impulsiveness and maladjustment) of two treatment groups (inpatient and outpatient) in which patients were assigned according to the clinical criteria of therapists; second, to identify which variables were more relevant in the therapeutic assignment of patients; third, to determine the differential progression of both treatment groups to evaluate the utility of the assignment criteria; and fourth, to compare the therapeutic progression between patients who met and did not meet the assignment criteria.

Journal of Substance Abuse Treatment, 2017, 76, 28-35 


\section{Methods}

The protocol for this study was approved by the ethics committees of the Universidad Pública de Navarra and the Fundación Proyecto Hombre de Navarra. Written informed consent was signed by all participants.

\subsection{Participants}

The initial sample consisted of 227 patients seeking treatment for addiction in the Proyecto Hombre de Navarra addiction treatment programme (Spain) from May 2010 to December 2012. This programme, which offers two modalities of intervention (outpatient and inpatient), is public and attends to patients from all over the region and who are representative of Spanish patients with addiction problems. Payment for treatment is not required. Every patient who consecutively attended the clinical centre was considered for study inclusion.

Study admission criteria included the following: a) meeting the diagnostic criteria for substance dependence disorder according to DSM-IV-TR (American Psychiatric Association, 2000), b) being between 18 and 65 years old, c) beginning the assigned treatment for drug-addiction, and d) giving consent to participation in the study. Following the above mentioned admission criteria, 46 people (20.3\%) were excluded from the study and 19 (8.4\%) refused to participate in the study. The reasons for exclusion were: a) not meeting DSM-IV-TR criteria for substance dependence (13 cases), b) refusing to receive treatment (13 cases), c) being derived to other service because of different reasons (11 cases), and d) being older that 65 (9 cases). Therefore, a total of 162 (71.4\% of total) subjects were studied.

\subsection{Instruments}

The instruments used in this study formed part of the clinical centre assessment package, and therefore they were not specifically selected for this research.

Journal of Substance Abuse Treatment, 2017, 76, 28-35 
The EuropASI (Kokkevi \& Hartgers, 1995) is the European version of the Addiction Severity Index scale (ASI) (McLellan, Luborsky, Woody, \& O’Brien, 1980). In this study, the Spanish version by Bobes, González, Sáiz, and Bousoño (1996) was employed. This interview assesses seven different areas: general medical condition, employment situation, alcohol consumption, use of other drugs, legal problems, family and social relationships, and psychological state. In this study, the Interviewer Severity Rating (ISR) was used. These ratings have shown their usefulness in different studies developed in treatment settings (López-Goñi, Fernández-Montalvo, \& Arteaga, 2012; López-Goñi et al., 2010). Although the Composite Scores provided by this instrument are usually used in the research field, in Spain the studies of López-Goñi et al. (2012) showed the utility of the ISRs for research purposes. Each area ranges from 0 (no problem) to 9 (extreme problem). The higher the score, the more need for treatment. The one-week test-retest reliabilities ranged from 0.67 to 0.96 in the seven different areas (González et al., 2002).

The Symptom Checklist (SCL-90-R) (Derogatis, 1992) is a self-report that assesses psychopathological symptoms. It is composed of 90 items, which are answered in a five-point Likert scale, from 0 (nothing) to 4 (extremely). It is comprised of nine primary symptom dimensions (somatisation, obsession-compulsion, interpersonal sensitivity, depression, anxiety, hostility, phobic anxiety, paranoid ideation, and psychoticism), and of three global indices of severity (the Global Severity Index (GSI), which reflects overall symptom severity, the Positive Symptom Distress Index (PSDI), which indicates symptom intensity, and the Positive Symptom Total (PST), which includes the number of items answered with a score different from 0). The internal consistency ranges from .70 to .90 . In this study, the percentiles of each dimension have been considered.

Journal of Substance Abuse Treatment, 2017, 76, 28-35 
The Barratt Impulsiveness Scale (BIS-10) (Barratt, 1985) aims to assess the degree of impulsivity of the subjects. It consists of 33 items scoring from 0 to 4 on a five-point Likert scale, and is composed of three factors having each one 11 items: motor, cognitive and non-planning impulsiveness. The total score ranges from 0 to 132 (the higher the score, the greater impulsiveness). The internal consistence is .84.

The Maladjustment Scale (Echeburúa, Corral, \& Fernández-Montalvo, 2000) reveals how each patient is affected in six different areas of everyday life (labour, social, leisure, partner, family and general). Each area ranges from 0 (nothing) to 5 (extremely) on a six-point Likert scale. The total scale range is 0-30. The higher the score, the higher the level of maladjustment. The internal consistency is .94.

\subsection{Treatment modalities}

Outpatient treatment. This is a cognitive-behavioural programme aimed at abstinence. The main therapeutic techniques are stimulus control, in vivo exposure and relapse prevention. Successful programme completion usually requires 12 months. The treatment includes weekly sessions (45-60 minutes) during the first 6 months, and biweekly sessions during the rest of time. The effectiveness of this programme in the addiction treatment has been proven (Fernández-Montalvo \& López-Goñi, 2010).

Inpatient treatment. This treatment comprises 2 therapeutic phases: residential therapeutic community and reinsertion. The first phase (therapeutic community), which has an estimated duration of 1 year, is inpatient-based and has 2 main goals: a) to develop or modify behaviours that will increase personal autonomy, and b) to learn coping skills to achieve relapse prevention. In this phase, group and occupational therapies are provided. The second phase (reinsertion), with a duration of approximately half a year, consists in a progressive reduction in the intensity of treatment. The goal of this phase is the reinsertion in social, family and employment areas through individual Journal of Substance Abuse Treatment, 2017, 76, 28-35 
and group therapies on an outpatient basis. Successful programme completion usually requires 18 months. The effectiveness of this programme in the addiction treatment has been proven (Fernández-Montalvo et al., 2008).

Both programmes take into account the patients’ family, involving the family members in the recovery process and giving them specific support to deal with the patient through specific support groups. Anyway, it is not compulsory the family participation to provide treatment to the patient.

\subsection{Experimental design}

A two-group experimental design (with two treatment groups for addiction) with repeated measures (pretreatment and 6-month follow-up) was used. The first group received outpatient treatment and the second group received inpatient (therapeutic community) intervention.

\subsection{Procedure}

All patients were interviewed and treated by clinical psychologists with ten or more years of experience in assessing and treating addictions.

The assessment of the sample was carried out in two sessions before beginning the treatment for addiction. Self-report questionnaires were administered with the presence and support of the interviewers. The sessions occurred once per week, and the time interval between sessions was the same for each participant. In the first session, data related to socio-demographic characteristics and drug consumption were assessed by the EuropASI. In the second session, questionnaires that assessed other variables (psychopathological symptoms, personality characteristics and maladjustment) were administered.

Once the clinical sample was assessed, patients were assigned to one of the two treatment modalities of the programme (outpatient or inpatient treatment) following the Journal of Substance Abuse Treatment, 2017, 76, 28-35 
usual criteria of the clinical centre. Generally, patients with severely high consumption and without family or partner support tended to receive the inpatient treatment. In contrast, patients with a better partner and/or family support structure usually received the outpatient treatment. These assignment criteria were based on clinical impressions of the therapeutic team.

The next evaluation, always in the format of a personal interview, took place at the 6-month follow-up after finishing the whole treatment, using the same instruments.

\subsection{Data Analysis}

Descriptive analyses were performed for all variables. In the bivariate analysis, $\chi^{2}$ or Student's $t$ test for independent samples were used depending on the nature of the variables analysed. Effect size (Cohen's $d$ or $w$ ) for all of the analyses were provided, taking into account Cohen's recommendation (Cohen, 1988): $d=0.20$ (small effect size), $d=0.50$ (medium effect size) and $d=0.80$ (large effect size). Regarding multivariate analysis, a Chi-squared Automatic Interaction Detection (CHAID) analysis with all the studied pretreatment variables was conducted to identify the main variables related to treatment assignment. This test evaluates the discriminant capacity of several independent variables over a dependent variable (in this study, assignment to inpatient or outpatient treatment), and it provides cut-offs for each variable defining the best-fit profile for both groups. Differences between groups in the effect size were calculated to establish which group presented a higher improvement. A difference of $p<.05$ was considered significant. All statistical analyses were performed using SPSS (vs. 23.0) software.

\section{Results}

\subsection{Characteristics of the sample}

Journal of Substance Abuse Treatment, 2017, 76, 28-35 
Of the 162 subjects studied, $73.5 \%$ were men $(n=119)$ and $26.5 \%$ were women $(n=43)$. Of this sample, $53.7 \%$ (87 patients) were assigned to inpatient treatment (therapeutic community). The rest of patients ( $n=75$; 46.3\%) received the outpatient programme.

The average age of the subjects was 36.4 years $(S D=8.9)$. The socioeconomic levels were middle to lower-middle class. The main substances that motivated treatment were cocaine and other stimulants (39.3\% of the sample) and alcohol (32.7\%). Other substances (e.g., heroin, cannabis, amphetamine, etc.) showed lower incidences but affected a total of $28 \%$ of the sample. Most of the subjects were single (63.8\%). Concerning education level, $47.9 \%$ had only primary studies, $44.7 \%$ had secondary studies, and 7.4\% had a university degree.

\subsection{Comparisons between groups in the pretreatment variables}

Regarding sociodemographic variables (Table 1), significant differences between groups were found in marital status and education level. Generally, patients in the therapeutic community presented with a higher rate of separation/divorce and with a lower educational level.

\section{PLACE TABLE 1 HERE}

Although inpatients showed greater alcohol consumption and outpatients greater cocaine consumption, there were no statistically significant differences between groups in terms of the substance that motivated their treatment (Table 1). However, as was expected, patients assigned to the therapeutic community scored significantly higher in most of the severity areas assessed by the EuropASI (Table 2). The effect sizes were large (above .80) in two areas: Family/Social and Medical.

PLACE TABLE 2 HERE

Journal of Substance Abuse Treatment, 2017, 76, 28-35 
From a psychopathological perspective, patients assigned to the therapeutic community tended to have a greater severity in the SCL-90-R, the Psychiatric area of the EuropASI and the BIS-10. Significant differences were found in depression (SCL90-R) and Psychiatry scale (EuropASI), but with a small effect size.

Finally, patients receiving an inpatient treatment in the therapeutic community showed statistically significant higher levels of maladjustment to everyday life in 4 out of the 6 areas assessed by the Maladjustment Scale. The effect sizes for these variables were small to medium.

\subsection{Objectifying patient placement criteria}

A CHAID analysis was conducted to find which variables were more relevant to becoming an objective criterion for the therapeutic assignment of patients. The results from this CHAID analysis showed that two variables (family/social support and partner maladjustment) were the most relevant (Figure 1).

\section{PLACE FIGURE 1 HERE}

Specifically, a score above 4 in the family/social support area of the EuropASI or in those cases with a score between 2 and 4 in the family/social area of EuropASI, a score above 2 in the partner subscale of the Maladjustment Scale correctly classified $73.5 \%$ of cases (96.6\% of inpatients and $46.7 \%$ of outpatients).

\subsection{Comparison between groups in therapeutic progression}

The results for repeated measurements analyses of the studied variables are shown in Table 3. Regarding inpatient treatment, the data showed that this group achieved statistically significant improvement in almost all the variables related to addiction severity, psychopathological symptoms, impulsiveness and maladjustment. Most of them presented medium to large effect sizes.

PLACE TABLE 3 HERE

Journal of Substance Abuse Treatment, 2017, 76, 28-35

http://dx.doi.org/10.1016/j.jsat.2017.02.014 
On the other hand, patients in outpatient treatment also achieved significant improvement in most of the variables studied. As in the inpatient group, the effect sizes for these variables were medium to large.

Once the progression of both treatment groups was analysed separately, differences between groups in the effect sizes were calculated to establish which group presented a higher improvement in the variables studied. The results showed that the improvement of both groups in psychopathological symptoms and impulsiveness was similar, although the effect sizes in the inpatient treatment group tended to be higher (Table 3). The main differences in the effect sizes were found in addiction severity (medical, alcohol, and family/social areas) and maladjustment (labour and family areas). In the rest of the variables, there were no relevant differences in the effect sizes.

\subsection{Differential therapeutic results depending on matching or mismatching the} assignment criteria

Several analyses were conducted to determine whether patients who were treated in the appropriate level of care according to the objective criteria obtained better outcomes than patients who were not. In the case of inpatient treatment, most of the patients (96.6\%) matched the obtained criteria and only 3.4\% of cases did not meet the criteria for inpatient treatment. In the case of outpatient treatment, the rate of patients who met the criteria was $46.7 \%$ and $53.3 \%$ did not. As there were only 3 patients mismatching the assignment criteria for inpatient treatment, the analyses were carried out only for outpatient treatment. The results obtained are shown in Table 4.

\section{PLACE TABLE 4 HERE}

Generally, the results showed that patients who met the criteria for outpatient treatment achieved small to medium effect sizes in most of the variables, and patients who did not meet the criteria achieved medium to large effect sizes. The repeatedJournal of Substance Abuse Treatment, 2017, 76, 28-35 
measures analyses indicated that patients who mismatched outpatient treatment (i.e. patients who actually met criteria for inpatient treatment) obtained greater statistically significant improvements in variables related to addiction severity, psychopathological symptoms, impulsiveness and maladjustment.

\section{Discussion}

In this naturalistic study, the profiles of patients receiving two different treatment modalities (inpatient or outpatient) have been compared to objectify the treatment assignment criteria used in a clinical centre. Due to the assignment method used by clinical professionals (based on their clinical perception), both groups of addictive patients presented significant differences between them in terms of sociodemographic, psychopathological and adjustment variables. After conducting statistical analyses, two objective variables supported the clinical assignment to both groups, family/social support (EuropASI) and partner maladjustment (Maladjustment Scale). This finding is relevant because it provides clinical professionals who work in addiction treatment centres with an objective criterion to make decisions about how to assign patients to the treatment modality that best fits them.

The two variables obtained as relevant assignment criteria support the results of previous studies showing that patients characterized by more severe substance use disorders, coexisting psychosocial problems, and weaker social and partner supports tended to be treated in inpatient programmes (Camilleri et al., 2012; Gregoire, 2000; Harrison \& Asche, 1999; Magor-Blatch et al., 2014; McGee \& MeeLee, 1997; McKay et al., 1997; Reif et al., 2014). Therefore, the psychosocial and partner consequences of the addiction problem become crucial variables when designing the therapeutic approach. The results of this study emphasize the need of following these objective matching criteria when clinicians assign patients to treatments, beyond the clinical Journal of Substance Abuse Treatment, 2017, 76, 28-35 
impression and the general idea that patients with higher addiction severity or worse psychiatric status should be treated in an inpatient setting.

On the other hand, in this study, the EuropASI has become a useful instrument to objectify clinical decisions about the treatment that better meets patient needs. The use of standardized assessments as methods for placement matching of patients with addiction problems has been shown to be useful in other studies (Camilleri et al., 2012; Rohrig et al., 2015). Regardless, the EuropASI assesses both family and social problems in one only area. The results obtained in this study support previous literature that indicated the relevance of assessing both dimensions separately to identify more accurately specific needs in these areas (López-Goñi et al., 2012).

The analysis of the therapeutic progression of patients belonging to both inpatient and outpatient treatment programmes has shown effect sizes that range from medium to large. Both treatment groups show statistically significant differences from the pretreatment to the follow-up assessment in most of the variables studied. Similar results have been obtained in previous studies that compare inpatient and outpatient treatment for patients with addiction problems (Harrison \& Asche, 1999). In this study, the patients who benefit most from both programmes are those presenting with a more severe addiction problem. These results are related directly to an important question in addiction treatment: what is the most cost-effective treatment? Although many studies comparing inpatient and outpatient treatment outcomes have been conducted (Harrison \& Asche, 1999; Magor-Blatch et al., 2014; McCarty et al., 2014; Miller \& Hester, 1986; Rohrig et al., 2015; Schmidt et al., 2009), no study to date has produced convincing evidence that treatment in residential settings is more effective than outpatient treatment.

Journal of Substance Abuse Treatment, 2017, 76, 28-35 
In this study, 53.3\% of the patients receiving outpatient treatment met the objective criteria obtained to receive inpatient treatment in the therapeutic community. Although they were mismatched into the outpatient treatment group, the effect sizes achieved were even greater than those obtained by patients who met the criteria for outpatient treatment. Previous studies with patient matching and mismatching the assignment criteria have also shown no differences between patients in the results obtained (McCarty et al., 2014; McKay et al., 1997). In this study, the good results obtained in the outpatient programme by patients who met the criteria for inpatient treatment questions the necessity of the inpatient treatment in these cases, as the outpatient treatment appears to be a sufficient level of care. These results should be taken into account because the use of inpatient hospital or residential settings contributes substantially to the cost of the treatment of patients with addiction problems.

This study has a number of limitations. First, one aspect that should be considered is the distribution of the sample by gender. Just a few women were included and, therefore, the conclusions of the study are mainly referred to male patients with addiction problems. Although this is a common circumstance in most of the studies about addictions, it should be taken into account when generalising the obtained results. A second limitation is related to the type of drug that motivated treatment. A larger sample size would allow the analysis of differential results depending on the type of substance used. Third, in this study, the motivation for seeking treatment has not been analysed. It would be interesting to include specific measures of treatment motivation in future studies. However, in this research the instruments used formed part of the clinical centre assessment package, and therefore they were not specifically selected for this study. On the other hand, some patients probably present a differential preference to receiving treatment in an outpatient or inpatient basis depending on their psychosocial Journal of Substance Abuse Treatment, 2017, 76, 28-35 
and family situation. It would be desirable to explore the influence of this differential preference on the results of both programmes. Finally, the inpatient programme studied in this research is a long-term therapeutic community. It may create a bias that prevents us from generalizing the results to other types of shorter stay residential treatments.

Regardless, the strengths of this research are related to its naturalistic design and to the use of standardized instruments to objectify the assignment criteria for drugaddicted patients. Being a naturalistic study, patients were not randomly assigned to a group but rather were placed into treatment based on clinical considerations. The establishment of objective patient placement criteria represents an approach to matching patients with addiction problems to the least expensive level of care needed to achieve treatment success. This is a necessary research topic due to shrinking treatment resources for addicted patients.

\section{Acknowledgements}

The authors would like to thank the "Proyecto Hombre de Navarra" programme staff for their help with developing this research.

\section{Funding}

This research did not receive any specific grant from funding agencies in the public, commercial or not-for-profit sectors.

\section{Declaration of interest}

The authors report no conflicts of interest. The authors alone are responsible for the content and writing of this paper.

Journal of Substance Abuse Treatment, 2017, 76, 28-35 
Journal of Substance Abuse Treatment, 2017, 76, 28-35

http://dx.doi.org/10.1016/j.jsat.2017.02.014 


\section{References}

American Psychiatric Association. (2000). Diagnostic and statistical manual of mental disorders (4th Ed. Rev.). Washington, D.C.: APA.

American Society of Addiction Medicine. (1996). Patient placement criteria for the treatment of substance-related disorder. Chevy Chase, MD: American Society of Addiction Medicine.

Barratt, E. S. (1985). Impulsiveness subtraits: Arousal and information procesing. In J. T. Spence \& C. E. Itard (Eds.), Motivation, Emotion and Personality. North Holland: Elsevier.

Bobes, J., González, M. P., Saiz, P. A., \& Bousoño, M. (1996). Índice Europeo de Severidad de la Adicción: EuropASI. Versión española [European Index of Severity of Addiction: EuropASI. Spanish Version]. Paper presented at the Actas de la IV Reunión Interregional de Psiquiatría, Gijón.

Camilleri, A. C., Cacciola, J. S., \& Jenson, M. R. (2012). Comparison of two ASI-based standardized patient placement approaches. Journal of Addictive Diseases, 31(2), 118-129. http://dx.doi.org/10.1080/10550887.2012.665727

Cohen, J. (1988). Statistical power analysis for the behavioral sciences. Hillsdale, New Jersey: Lawrence Erlbaum Associates.

Derogatis, L. R. (1992). The SCL-90-R. Baltimore: Clinical Psychometric Research. Echeburúa, E., Corral, P., \& Fernández-Montalvo, J. (2000). Escala de Inadaptación (EI): propiedades psicométricas en contextos clínicos. Análisis y Modificación de Conducta, 26(107), 325-340.

Journal of Substance Abuse Treatment, 2017, 76, 28-35 
Fernández-Montalvo, J., \& López-Goñi, J. J. (2010). Comparison of completers and dropouts in psychological treatment for cocaine addiction. Addiction Research \& Theory, 18(4), 433-441. http://dx.doi.org/10.3109/16066350903324826

Fernández-Montalvo, J., López-Goñi, J. J., Illescas, C., Landa, N., \& Lorea, I. (2008). Evaluation of a therapeutic community treatment program: A long-term followup study in Spain. Substance Use \& Misuse, 43(10), 1362-1377.

González, F., Salvador, L., Martínez, J. M., López, A., Ruz, I., \& Guerra, D. (2002). Estudio de fiabilidad y validez de la versión española de la entrevista clínica Addiction Severity Index (ASI) Instrumentos de evaluación en drogodependencias. Madrid: Aula Médica.

Gregoire, T. K. (2000). Factors associated with level of care assignment in substance abuse treatment. Journal of Substance Abuse Treatment, 18(3), 241-248. http://dx.doi.org/10.1016/s0740-5472(99)00053-7

Harrison, P. A., \& Asche, S. E. (1999). Comparison of substance abuse treatment outcomes for inpatients and outpatients. Journal of Substance Abuse Treatment, 17(3), 207-220. http://dx.doi.org/10.1016/s0740-5472(99)00004-5

Hoffman, N. G., Halikas, J. A., Mee-Lee, D., \& Weedman, R. (1991). Patient Placement Criteria for the Treatment of Psychoactive Substance Use Disorders (PPC-1). Washington, DC: American Society of Addiction Medicine.

Hubbard, R. L., Craddock, S. G., \& Anderson, J. (2003). Overview of 5-year followup outcomes in the drug abuse treatment outcome studies (DATOS). Journal of Substance Abuse Treatment, 25(3), 125-134. http://dx.doi.org/10.1016/s07405472(03)00130-2

Journal of Substance Abuse Treatment, 2017, 76, 28-35 
Kokkevi, A., \& Hartgers, C. (1995). European adaptation of a multidimensional assessment instrument for drug and alcohol dependence. European Addiction Research, 1, 208-210.

López-Goñi, J. J., Fernández-Montalvo, J., \& Arteaga, A. (2012). Predictive validity of the EuropASI: Clinical diagnosis or composite scoring? Journal of Substance Abuse Treatment, 42(4), 392-399. http://dx.doi.org/10.1016/j.jsat.2011.09.011

López-Goñi, J. J., Fernández-Montalvo, J., Cacho, R., \& Arteaga, A. (2014). Profile of addicted patients who reenter treatment programs. Substance Abuse, 35(2), 176183. http://dx.doi.org/10.1080/08897077.2013.826614

López-Goñi, J. J., Fernández-Montalvo, J., Menéndez, J. C., Yudego, F., García, A., \& Esarte, S. (2010). Group and individual change in the treatment of drug addictions: A follow-up study in Therapeutic Communities. Spanish Journal of Psychology, 13(2), 906-913.

Magor-Blatch, L., Bhullar, N., Thomson, B., Thorsteinsson, E. (2014). A systematic review of studies examining effectiveness of therapeutic communities. Therapeutic Communities: The International Journal of Therapeutic Communities 35(4), 168-184. http://dx.doi.org/10.1108/TC-07-2013-0024

McCarty, D., Braude, L., Lyman, D. R., Dougherty, R. H., Daniels, A. S., Ghose, S. S., \& Delphin-Rittmon, M. E. (2014). Substance Abuse Intensive Outpatient Programs: Assessing the Evidence. Psychiatric Services (Washington, D.C.), 65(6), 718-726. http://doi.org/10.1176/appi.ps.201300249

McGee, M. D., \& MeeLee, D. (1997). Rethinking patient placement: The Human Service Matrix model for matching services to needs. Journal of Substance Abuse Treatment, 14(2), 141-148. http://dx.doi.org/10.1016/s07405472(96)00122-5

Journal of Substance Abuse Treatment, 2017, 76, 28-35 http://dx.doi.org/10.1016/j.jsat.2017.02.014 
McKay, J. R., Cacciola, J. S., McLellan, A. T., Alterman, A. I., \& Wirtz, P. W. (1997). An initial evaluation of the psychosocial dimensions of the American Society of Addiction Medicine Criteria for inpatient versus intensive outpatient substance abuse rehabilitation. Journal of Studies on Alcohol, 58(3), 239-252.

McLellan, A. T., Luborsky, L., Woody, G. E., \& O’Brien, C. P. (1980). An improved diagnostic evaluation instrument for substance abuse patients: The Addiction Severity Index. The Journal of Nervous and Mental Disease(168), 26-33.

Miller, W. R., \& Hester, R. K. (1986). Inpatient alcoholism treatment. Who benefits? American Psychologist, 41(7), 794-805. http://dx.doi.org/10.1037//0003066x.41.7.794

Reif, S., George, P., Braude, L., Dougherty, R. H., Daniels, A. S., Ghose, S. S., \& Delphin-Rittmon, M. E. (2014). Residential treatment for individuals with substance use disorders: Assessing the evidence. Psychiatric Services (Washington, D.C.), 65(3), 301-312. http://dx.doi.org/10.1176/appi.ps.201300242

Rohrig, J., Buchholz, A., Wahl, S., \& Berner, M. (2015). Placement matching for patients with alcohol use disorders using standardized assessment: a pilot study. Journal of Substance Use, 20(2), 97-105. http://dx.doi.org/10.3109/14659891.2013.866175

Stallvik, M., Gastfriend, D. R., \& Nordhal, H. M. (2015). Matching patients with substance use disorder to optimal level of care with the ASAM Criteria software. Journal of Substance Use, 20(6), 389-398. http://dx.doi.org/10.3109/14659891.2014.934305

Schmidt, P., Kufner, H., Lohnert, B., Kolb, W., Zemlin, U., \& Soyka, M. (2009). Efficiency of Outpatient and Inpatient Alcohol Treatment - Predictors of Journal of Substance Abuse Treatment, 2017, 76, 28-35 http://dx.doi.org/10.1016/j.jsat.2017.02.014 
Outcome. Fortschritte Der Neurologie Psychiatrie, 77(8), 451-456.

http://dx.doi.org/10.1055/s-0028-1109501

Vanderplasschen, W., Colpaert, K., Autrique, M., Rapp, R. C., Pearce, S., Broekaert, E., \& Vandevelde, S. (2013). Therapeutic Communities for Addictions: A Review of Their Effectiveness from a Recovery-Oriented Perspective. The Scientific World Journal, 2013. http://dx.doi.org/10.1155/2013/427817

Wallace, A. E., \& Weeks, W. B. (2004). Substance abuse intensive outpatient treatment: Does program graduation matter? Journal of Substance Abuse Treatment, 27(1), 27-30. http://dx.doi.org/10.1016/j.jsat.2004.03.006 
Table 1

Comparisons of socio-demographic and consumption variables

\begin{tabular}{|c|c|c|c|c|c|c|c|c|c|}
\hline & \multicolumn{2}{|c|}{$\begin{array}{c}\text { Total } \\
(\mathrm{N}=162)\end{array}$} & \multicolumn{2}{|c|}{$\begin{array}{l}\text { Therapeutic } \\
\text { community } \\
(\mathbf{n}=\mathbf{8 7})\end{array}$} & \multicolumn{2}{|c|}{$\begin{array}{c}\text { Outpatient } \\
\text { treatment } \\
(n=75)\end{array}$} & \multirow[b]{2}{*}{ t } & \multirow[b]{2}{*}{$\mathbf{p}$} & \multirow[b]{2}{*}{ d } \\
\hline & Mean & SD & Mean & SD & Mean & SD & & & \\
\hline \multirow[t]{2}{*}{ Age } & 36.4 & 8.9 & 37.3 & 9.1 & 35.2 & 8.5 & 1.4145 & .160 & 0.23 \\
\hline & $\mathbf{N}$ & (\%) & $\mathbf{n}$ & $(\%)$ & $\mathbf{n}$ & $(\%)$ & $\begin{array}{ll}X^{2} & \text { df } \\
\end{array}$ & $\mathbf{p}$ & $\mathbf{w}$ \\
\hline \multicolumn{10}{|l|}{ Gender } \\
\hline Men & 119 & $73.5 \%$ & 63 & $72.4 \%$ & 56 & $74.7 \%$ & \multirow{2}{*}{0.11} & \multirow{2}{*}{.746} & \multirow{2}{*}{0.06} \\
\hline Women & 43 & $26.5 \%$ & 24 & $27.6 \%$ & 19 & $25.3 \%$ & & & \\
\hline \multicolumn{10}{|l|}{ Marital status } \\
\hline Single & 97 & $63.8 \%$ & 48 & $59.3 \%$ & 49 & $69.0 \%$ & \multirow{3}{*}{8.92} & \multirow{3}{*}{.012} & \multirow{3}{*}{0.43} \\
\hline Married & 30 & $19.7 \%$ & 13 & $16.0 \%$ & 17 & $23.9 \%$ & & & \\
\hline Separated/Divorced & 25 & $16.5 \%$ & 20 & $24.7 \%$ & 5 & $7.0 \%$ & & & \\
\hline \multicolumn{10}{|l|}{ Education level } \\
\hline Primary & 58 & $47.9 \%$ & 35 & $59.3 \%$ & 23 & $37.1 \%$ & \multirow{3}{*}{7.32} & \multirow{3}{*}{.026} & \multirow{3}{*}{0.51} \\
\hline Secondary & 54 & $44.7 \%$ & 19 & $32.2 \%$ & 35 & $56.5 \%$ & & & \\
\hline University & 9 & $7.4 \%$ & 5 & $8.5 \%$ & 4 & $6.5 \%$ & & & \\
\hline \multicolumn{10}{|c|}{$\begin{array}{l}\text { Substance motivating } \\
\text { treatment }\end{array}$} \\
\hline Alcohol & 49 & $32.7 \%$ & 31 & $38.3 \%$ & 18 & $26.1 \%$ & \multirow{3}{*}{2.72} & \multirow{3}{*}{.252} & \multirow{3}{*}{0.27} \\
\hline Cocaine & 59 & $39.3 \%$ & 28 & $34.6 \%$ & 31 & $44.9 \%$ & & & \\
\hline Other & 42 & $28.0 \%$ & 22 & $27.1 \%$ & 20 & $28.9 \%$ & & & \\
\hline
\end{tabular}

Journal of Substance Abuse Treatment, 2017, 76, 28-35

http://dx.doi.org/10.1016/j.jsat.2017.02.014 
Table 2

Comparisons of severity of addiction, psychopathological symptoms, personality characteristics and maladjustment variables

\begin{tabular}{|c|c|c|c|c|c|c|c|c|c|c|}
\hline & \multicolumn{2}{|c|}{$\begin{array}{c}\text { Total } \\
\mathrm{N}=162 \\
\end{array}$} & \multicolumn{2}{|c|}{$\begin{array}{l}\text { Therapeutic } \\
\text { community } \\
(\mathbf{n}=\mathbf{8 7})\end{array}$} & \multicolumn{2}{|c|}{$\begin{array}{c}\text { Outpatient } \\
\text { treatment } \\
(\mathbf{n}=75)\end{array}$} & \multirow[b]{2}{*}{ t } & \multirow[b]{2}{*}{ df } & \multirow[b]{2}{*}{$\mathbf{p}$} & \multirow[b]{2}{*}{ d } \\
\hline & $\mathbf{M}$ & SD & $\mathbf{M}$ & SD & $\mathbf{M}$ & SD & & & & \\
\hline \multicolumn{11}{|l|}{ EuropASI } \\
\hline Medical & 2.83 & 1.83 & 3.56 & 1.97 & 2.00 & 1.21 & 5.8 & 150 & $<.001$ & 0.85 \\
\hline Employment/Support & 3.61 & 1.94 & 4.28 & 1.93 & 2.85 & 1.67 & 4.9 & 150 & $<.001$ & 0.74 \\
\hline Alcohol & 4.39 & 2.17 & 5.01 & 2.15 & 3.67 & 1.96 & 4.0 & 149 & $<.001$ & 0.62 \\
\hline Use of other drugs & 4.44 & 2.06 & 4.69 & 2.38 & 4.14 & 1.59 & 1.6 & 149 & .103 & 0.27 \\
\hline Legal & 2.64 & 1.99 & 2.72 & 2.19 & 2.56 & 1.74 & 0.5 & 150 & .638 & 0.08 \\
\hline Family/Social & 4.67 & 1.85 & 5.53 & 1.67 & 3.69 & 1.55 & 7.0 & 150 & $<.001$ & 0.99 \\
\hline Psychiatric & 4.27 & 1.71 & 4.59 & 1.72 & 3.90 & 1.64 & 2.5 & 150 & .012 & 0.40 \\
\hline \multicolumn{11}{|l|}{ SCL-90-R } \\
\hline Global Severity Index & 70.0 & 31.5 & 74.67 & 28.48 & 64.61 & 34.09 & 1.9 & 123.3 & .065 & 0.32 \\
\hline $\begin{array}{l}\text { Positive Symptom Distress } \\
\text { Index }\end{array}$ & 49.9 & 30.2 & 52.70 & 30.80 & 46.61 & 29.40 & 1.2 & 135 & .240 & 0.20 \\
\hline Positive Symptom Total & 74.0 & 29.3 & 77.62 & 26.41 & 69.95 & 32.01 & 1.5 & 135 & .127 & 0.26 \\
\hline Somatisation & 62.4 & 31.4 & 64.58 & 29.75 & 60.55 & 33.41 & 0.7 & 135 & .457 & 0.13 \\
\hline Obsession-compulsion & 64.1 & 32.5 & 65.86 & 30.89 & 62.72 & 34.65 & 0.6 & 135 & .575 & 0.10 \\
\hline Interpersonal sensitivity & 68.2 & 31.1 & 73.29 & 28.95 & 62.84 & 32.93 & 2.0 & 126.5 & .052 & 0.33 \\
\hline Depression & 67.9 & 30.5 & 73.97 & 25.77 & 61.17 & 34.27 & 2.4 & 116.0 & .016 & 0.42 \\
\hline Anxiety & 64.7 & 32.9 & 69.99 & 29.88 & 59.16 & 35.61 & 1.9 & 123.6 & .058 & 0.33 \\
\hline Hostility & 54.0 & 33.0 & 57.44 & 31.92 & 50.05 & 34.07 & 1.3 & 135 & .192 & 0.22 \\
\hline Phobic anxiety & 52.0 & 39.1 & 55.01 & 38.32 & 48.84 & 40.20 & 0.9 & 135 & .360 & 0.16 \\
\hline Paranoid ideation & 67.7 & 32.2 & 72.12 & 30.09 & 63.27 & 34.24 & 1.6 & 135 & .109 & 0.27 \\
\hline Psychoticism & 71.2 & 30.1 & 75.40 & 28.63 & 66.64 & 31.50 & 1.7 & 135 & .091 & 0.29 \\
\hline \multicolumn{11}{|l|}{ BIS } \\
\hline Motor impulsiveness & 18.93 & 8.04 & 19.52 & 7.79 & 18.24 & 8.33 & 1.0 & 160 & .315 & 0.16 \\
\hline Attentional impulsiveness & 21.33 & 6.14 & 21.48 & 6.21 & 21.16 & 6.10 & 0.3 & 160 & .740 & 0.05 \\
\hline Non-planning impulsiveness & 17.81 & 6.76 & 18.75 & 6.64 & 16.73 & 6.78 & 1.9 & 160 & .058 & 0.30 \\
\hline Total impulsiveness & 58.07 & 17.25 & 59.75 & 17.25 & 56.13 & 17.16 & 1.3 & 160 & .185 & 0.21 \\
\hline \multicolumn{11}{|l|}{ Maladjustment } \\
\hline Labour & 3.07 & 1.76 & 3.37 & 1.59 & 2.72 & 1.88 & 2.4 & 160.0 & .019 & 0.37 \\
\hline Social & 3.17 & 1.50 & 3.38 & 1.33 & 2.92 & 1.64 & 2.0 & 160.0 & .051 & 0.31 \\
\hline Leisure & 3.23 & 1.47 & 3.39 & 1.31 & 3.04 & 1.63 & 1.5 & 141.3 & .137 & 0.24 \\
\hline Partner & 3.28 & 1.64 & 3.59 & 1.39 & 2.92 & 1.84 & 2.6 & 132.4 & .012 & 0.41 \\
\hline Family & 3.29 & 1.43 & 3.74 & 1.15 & 2.77 & 1.55 & 4.4 & 132.4 & $<.001$ & 0.68 \\
\hline General & 3.75 & 1.25 & 4.01 & 1.06 & 3.45 & 1.38 & 2.9 & 136.0 & .005 & 0.45 \\
\hline Total maladjustment & 19.70 & 7.23 & 21.47 & 6.19 & 17.65 & 7.83 & 3.4 & 140.2 & .001 & 0.53 \\
\hline
\end{tabular}

$\mathrm{SD}=$ standard deviation; $\mathrm{df}=$ degree of freedom; $\mathrm{d}=$ Cohen's $\mathrm{d}$

Journal of Substance Abuse Treatment, 2017, 76, 28-35

http://dx.doi.org/10.1016/j.jsat.2017.02.014 
Table 3

Results of the repeated-measures analyses (pretreatment and 6-month follow-up) and effect size

\begin{tabular}{|c|c|c|c|c|c|c|c|c|c|c|c|c|c|c|c|c|c|}
\hline & \multicolumn{8}{|c|}{ Inpatient treatment } & \multicolumn{9}{|c|}{ Outpatient treatment } \\
\hline & \multicolumn{2}{|c|}{ Pretreatment } & \multicolumn{2}{|c|}{ Follow-up } & \multirow[b]{2}{*}{$\mathbf{t}$} & \multirow[b]{2}{*}{ df } & \multirow[b]{2}{*}{$\mathbf{p}$} & \multirow[b]{2}{*}{ d } & \multicolumn{2}{|c|}{ Pretreatment } & \multicolumn{2}{|c|}{ Follow-up } & \multirow[b]{2}{*}{$\mathbf{t}$} & \multirow[b]{2}{*}{ df } & \multirow[b]{2}{*}{$\mathbf{p}$} & \multirow[b]{2}{*}{ d } & \multirow[b]{2}{*}{ d diff. } \\
\hline & $\mathbf{M}$ & SD & $\mathbf{M}$ & SD & & & & & $\mathbf{M}$ & SD & $\mathbf{M}$ & SD & & & & & \\
\hline \multicolumn{18}{|l|}{ EuropASI } \\
\hline Medical & 3.74 & 1.60 & 1.94 & 1.87 & 4.3 & 33 & $<.001$ & 0.96 & 1.77 & 0.99 & 1.52 & 1.48 & 1.2 & 30 & .223 & 0.17 & 0.79 \\
\hline Employment/Support & 4.85 & 1.67 & 5.18 & 2.44 & 0.8 & 33 & .422 & -0.13 & 2.42 & 1.50 & 2.87 & 2.74 & 1.1 & 30 & .260 & -0.16 & 0.03 \\
\hline Alcohol & 5.85 & 1.83 & 3.21 & 2.33 & 5.3 & 33 & $<.001$ & 1.13 & 3.81 & 1.89 & 2.23 & 2.20 & 3.6 & 30 & .001 & 0.72 & 0.41 \\
\hline Use of other drugs & 4.53 & 2.53 & 2.44 & 2.71 & 5.2 & 33 & $<.001$ & 0.77 & 3.87 & 1.59 & 2.13 & 2.28 & 4.5 & 30 & $<.001$ & 0.77 & 0.00 \\
\hline Legal & 2.74 & 2.45 & 1.68 & 2.50 & 3.3 & 33 & .002 & 0.42 & 2.26 & 1.50 & 1.23 & 1.78 & 3.6 & 30 & .001 & 0.58 & -0.16 \\
\hline Family/Social & 6.00 & 1.58 & 3.56 & 1.88 & 7.9 & 33 & $<.001$ & 1.30 & 3.48 & 1.43 & 3.06 & 1.67 & 1.2 & 30 & .235 & 0.25 & 1.05 \\
\hline Psychiatric & 4.82 & 1.55 & 3.09 & 2.57 & 4.1 & 33 & $<.001$ & 0.67 & 3.97 & 1.49 & 2.26 & 2.13 & 5.1 & 30 & $<.001$ & 0.80 & -0.13 \\
\hline \multicolumn{18}{|l|}{ SCL-90-R } \\
\hline Global Severity Index & 75.13 & 30.27 & 53.35 & 38.80 & 4.6 & 47 & $<.001$ & 0.56 & 65.09 & 34.05 & 51.30 & 37.86 & 2.6 & 42 & .013 & 0.36 & 0.2 \\
\hline Positive Symptom Distress Index & 50.21 & 31.72 & 42.31 & 32.70 & 1.8 & 47 & .071 & 0.24 & 47.70 & 30.09 & 37.53 & 31.08 & 2.1 & 42 & .045 & 0.33 & -0.09 \\
\hline Positive Symptom Total & 78.92 & 28.42 & 58.19 & 38.10 & 4.6 & 47 & $<.001$ & 0.54 & 70.86 & 31.63 & 55.72 & 37.59 & 2.9 & 42 & .006 & 0.40 & 0.14 \\
\hline Somatisation & 64.00 & 31.74 & 48.00 & 36.30 & 3.8 & 47 & $<.001$ & 0.44 & 59.56 & 33.75 & 52.98 & 33.07 & 1.4 & 42 & .182 & 0.20 & 0.24 \\
\hline Obsession-compulsion & 66.29 & 31.84 & 51.56 & 36.65 & 3.5 & 47 & .001 & 0.40 & 66.72 & 34.11 & 50.60 & 36.51 & 3.4 & 42 & .001 & 0.44 & -0.04 \\
\hline Interpersonal sensitivity & 74.50 & 29.93 & 55.90 & 34.51 & 4.0 & 47 & $<.001$ & 0.54 & 64.47 & 32.72 & 55.09 & 35.64 & 1.9 & 42 & .070 & 0.26 & 0.28 \\
\hline Depression & 75.27 & 25.52 & 55.69 & 35.90 & 4.1 & 47 & $<.001$ & 0.55 & 62.07 & 34.39 & 50.09 & 36.44 & 2.3 & 42 & .026 & 0.33 & 0.22 \\
\hline Anxiety & 70.65 & 29.64 & 51.13 & 37.08 & 3.8 & 47 & $<.001$ & 0.53 & 60.19 & 36.56 & 45.72 & 34.84 & 2.4 & 42 & .019 & 0.42 & 0.11 \\
\hline Hostility & 60.56 & 30.10 & 42.33 & 35.66 & 4.2 & 47 & $<.001$ & 0.51 & 50.05 & 34.31 & 35.26 & 32.93 & 2.4 & 42 & .019 & 0.45 & 0.06 \\
\hline Phobic anxiety & 51.21 & 41.70 & 44.23 & 38.51 & 1.0 & 47 & .308 & 0.18 & 48.19 & 42.57 & 40.14 & 40.24 & 1.4 & 42 & .157 & 0.20 & -0.02 \\
\hline Paranoid ideation & 76.52 & 26.57 & 60.50 & 36.22 & 3.4 & 47 & .002 & 0.44 & 64.02 & 35.50 & 54.42 & 33.07 & 1.9 & 42 & .065 & 0.29 & 0.15 \\
\hline Psychoticism & 76.06 & 29.58 & 60.27 & 38.12 & 3.3 & 47 & .002 & 0.41 & 68.09 & 31.42 & 52.65 & 39.81 & 2.3 & 42 & .028 & 0.39 & 0.02 \\
\hline \multicolumn{18}{|l|}{ BIS } \\
\hline Motor impulsiveness & 20.17 & 7.68 & 17.67 & 7.83 & 2.3 & 53 & .023 & 0.32 & 18.04 & 8.57 & 17.06 & 6.81 & 0.9 & 46 & .356 & 0.14 & 0.18 \\
\hline Attentional impulsiveness & 21.20 & 6.49 & 19.74 & 5.87 & 1.8 & 53 & .070 & 0.25 & 20.32 & 6.13 & 19.77 & 5.21 & 0.8 & 46 & .452 & 0.11 & 0.14 \\
\hline Non-planning impulsiveness & 18.83 & 6.72 & 15.80 & 7.74 & 3.3 & 53 & .001 & 0.39 & 15.98 & 5.87 & 15.36 & 7.35 & 0.7 & 46 & .458 & 0.08 & 0.31 \\
\hline Total impulsiveness & 60.20 & 17.66 & 53.20 & 19.11 & 3.3 & 53 & .001 & 0.37 & 54.34 & 16.54 & 52.19 & 16.67 & 1.1 & 46 & .264 & 0.13 & 0.24 \\
\hline \multicolumn{18}{|l|}{ Maladjustment } \\
\hline Labour & 3.54 & 1.48 & 1.61 & 1.64 & 6.7 & 53 & $<.001$ & 1.17 & 2.74 & 1.85 & 1.64 & 1.58 & 4.0 & 46 & $<.001$ & 0.70 & 0.47 \\
\hline
\end{tabular}

Journal of Substance Abuse Treatment, 2017, 76, 28-35 


\begin{tabular}{|c|c|c|c|c|c|c|c|c|c|c|c|c|c|c|c|c|c|}
\hline Social & 3.41 & 1.49 & 1.78 & 1.61 & 6.6 & 53 & $<.001$ & 1.01 & 3.06 & 1.69 & 1.83 & 1.62 & 4.3 & 46 & $<.001$ & 0.76 & 0.25 \\
\hline Leisure & 3.37 & 1.38 & 1.80 & 1.66 & 6.7 & 53 & $<.001$ & 0.95 & 3.11 & 1.66 & 1.91 & 1.49 & 4.8 & 46 & $<.001$ & 0.80 & 0.15 \\
\hline Partner & 3.63 & 1.35 & 1.85 & 1.71 & 6.8 & 53 & $<.001$ & 1.04 & 3.23 & 1.87 & 1.81 & 1.70 & 4.3 & 46 & $<.001$ & 0.84 & 0.2 \\
\hline Family & 3.78 & 1.08 & 1.72 & 1.51 & 8.2 & 53 & $<.001$ & 1.36 & 2.79 & 1.56 & 1.53 & 1.53 & 3.9 & 46 & $<.001$ & 0.82 & 0.54 \\
\hline General & 3.98 & 1.07 & 2.00 & 1.63 & 8.7 & 53 & $<.001$ & 1.22 & 3.53 & 1.41 & 2.06 & 1.69 & 5.3 & 46 & $<.001$ & 0.87 & 0.35 \\
\hline Total maladjustment & 21.70 & 5.93 & 10.76 & 8.22 & 9.2 & 53 & $<.001$ & 1.33 & 18.47 & 7.99 & 10.79 & 8.00 & 5.5 & 46 & $<.001$ & 0.96 & 0.37 \\
\hline
\end{tabular}

$\mathrm{SD}$ = standard deviation; $\mathrm{df}=$ degree of freedom; $\mathrm{d}=$ Cohen's $\mathrm{d}$

Journal of Substance Abuse Treatment, 2017, 76, 28-35

http://dx.doi.org/10.1016/j.jsat.2017.02.014 
Table 4

Results of the repeated-measures analyses (pretreatment and 6-month follow-up) and effect size (outpatients)

\begin{tabular}{|c|c|c|c|c|c|c|c|c|c|c|c|c|c|c|c|c|c|}
\hline & \multicolumn{8}{|c|}{ Matching criteria } & \multicolumn{8}{|c|}{ Mismatching criteria } & \multirow[b]{3}{*}{ d diff. } \\
\hline & \multicolumn{2}{|c|}{ Pretreatment } & \multicolumn{2}{|c|}{ Follow-up } & \multirow[b]{2}{*}{$\mathbf{t}$} & \multirow[b]{2}{*}{ df } & \multirow[b]{2}{*}{$\mathbf{p}$} & \multirow[b]{2}{*}{ d } & \multicolumn{2}{|c|}{ Pretreatment } & \multicolumn{2}{|c|}{ Follow-up } & \multirow[b]{2}{*}{$\mathbf{t}$} & \multirow[b]{2}{*}{ df } & \multirow[b]{2}{*}{$\mathbf{p}$} & \multirow[b]{2}{*}{ d } & \\
\hline & $\mathbf{M}$ & SD & $\mathbf{M}$ & SD & & & & & $\mathbf{M}$ & SD & $\mathbf{M}$ & SD & & & & & \\
\hline \multicolumn{18}{|l|}{ EuropASI } \\
\hline Medical & 1.64 & 1.15 & 1.36 & 1.44 & 1.1 & 13 & .302 & 0.24 & 1.88 & 0.85 & 1.65 & 1.53 & 7.4 & 16 & .466 & 0.27 & -0.03 \\
\hline Employment/Support & 2.21 & 1.37 & 2.36 & 2.62 & 0.2 & 13 & .789 & -0.10 & 2.59 & 1.62 & 3.29 & 2.84 & 1.2 & 16 & .241 & -0.43 & 0.33 \\
\hline Alcohol & 3.71 & 2.12 & 2.14 & 1.56 & 2.4 & 13 & .032 & 0.74 & 3.88 & 1.72 & 2.29 & 2.66 & 2.6 & 16 & .018 & 0.92 & -0.18 \\
\hline Use of other drugs & 3.57 & 1.65 & 2.86 & 2.62 & 1.2 & 13 & .253 & 0.43 & 4.12 & 1.53 & 1.53 & 1.80 & 6.1 & 16 & $<.001$ & 1.69 & -1.26 \\
\hline Legal & 1.79 & 1.12 & 1.21 & 1.71 & 1.1 & 13 & .263 & 0.51 & 2.65 & 1.69 & 1.24 & 1.88 & 4.5 & 16 & $<.001$ & 0.83 & -0.32 \\
\hline Family/Social & 2.43 & 0.93 & 2.93 & 1.63 & 1.3 & 13 & .221 & -0.53 & 4.35 & 1.17 & 3.18 & 1.74 & 2.4 & 16 & .026 & 1.00 & -1.53 \\
\hline Psychiatric & 3.21 & 1.42 & 2.07 & 1.73 & 3.3 & 13 & .006 & 0.80 & 4.59 & 1.27 & 2.41 & 2.45 & 4.1 & 16 & .001 & 1.71 & -0.91 \\
\hline \multicolumn{18}{|l|}{ SCL-90-R } \\
\hline Global Severity Index & 57.18 & 31.74 & 50.82 & 40.64 & 0.6 & 16 & .503 & 0.20 & 70.27 & 35.09 & 51.61 & 36.75 & 2.9 & 25 & .007 & 0.53 & -0.33 \\
\hline Positive Symptom Distress Index & 38.18 & 30.64 & 32.64 & 29.96 & 0.7 & 16 & .453 & 0.18 & 53.92 & 28.62 & 40.73 & 31.95 & 1.9 & 25 & .060 & 0.46 & -0.28 \\
\hline Positive Symptom Total & 67.29 & 29.99 & 56.23 & 40.47 & 1.1 & 16 & .271 & 0.36 & 73.19 & 33.02 & 55.38 & 36.39 & 2.9 & 25 & .007 & 0.53 & -0.17 \\
\hline Somatisation & 48.12 & 34.12 & 44.00 & 36.15 & 0.5 & 16 & .637 & 0.12 & 67.04 & 31.95 & 58.84 & 30.16 & 1.4 & 25 & .175 & 0.25 & -0.13 \\
\hline Obsession-compulsion & 57.88 & 35.88 & 50.82 & 36.48 & 0.8 & 16 & .394 & 0.19 & 72.50 & 32.30 & 50.46 & 37.24 & 3.9 & 25 & .001 & 0.68 & -0.49 \\
\hline Interpersonal sensitivity & 51.88 & 31.20 & 48.17 & 41.24 & 0.3 & 16 & .717 & 0.11 & 72.69 & 31.57 & 59.61 & 31.49 & 2.5 & 25 & .018 & 0.41 & -0.3 \\
\hline Depression & 50.71 & 34.35 & 46.29 & 37.48 & 0.5 & 16 & .636 & 0.12 & 69.50 & 32.96 & 52.57 & 36.27 & 2.8 & 25 & .010 & 0.51 & -0.39 \\
\hline Anxiety & 55.41 & 33.98 & 47.58 & 38.19 & 0.7 & 16 & .469 & 0.23 & 63.31 & 38.47 & 44.50 & 33.18 & 2.6 & 25 & .013 & 0.48 & -0.25 \\
\hline Hostility & 41.59 & 33.08 & 39.11 & 36.20 & 0.2 & 16 & .817 & 0.07 & 55.58 & 34.59 & 32.73 & 31.08 & 3.2 & 25 & .003 & 0.66 & -0.59 \\
\hline Phobic anxiety & 41.12 & 42.05 & 49.35 & 43.59 & 1.0 & 16 & .332 & -0.19 & 52.81 & 43.08 & 34.11 & 37.53 & 2.7 & 25 & .012 & 0.43 & -0.62 \\
\hline Paranoid ideation & 59.47 & 33.49 & 55.82 & 33.71 & 0.4 & 16 & 688 & 0.10 & 67.00 & 37.09 & 53.50 & 33.28 & 2.2 & 25 & .034 & 0.36 & -0.26 \\
\hline Psychoticism & 67.18 & 24.06 & 56.05 & 41.08 & 1.0 & 16 & .308 & 0.46 & 68.69 & 35.88 & 50.42 & 39.61 & 2.0 & 25 & .052 & 0.50 & -0.04 \\
\hline \multicolumn{18}{|l|}{ BIS } \\
\hline Motor impulsiveness & 17.83 & 8.23 & 19.05 & 6.44 & 0.7 & 17 & .459 & -0.14 & 18.17 & 8.92 & 15.82 & 6.84 & 1.7 & 28 & .090 & 0.26 & -0.4 \\
\hline Attentional impulsiveness & 21.06 & 5.62 & 21.55 & 5.41 & 0.4 & 17 & .676 & -0.08 & 19.86 & 6.47 & 18.65 & 4.84 & 1.3 & 28 & .203 & 0.18 & -0.26 \\
\hline Non-planning impulsiveness & 16.67 & 6.26 & 17.72 & 7.27 & 0.8 & 17 & .435 & -0.16 & 15.55 & 5.68 & 13.89 & 7.13 & 1.6 & 28 & .118 & 0.29 & -0.45 \\
\hline Total impulsiveness & 55.56 & 16.67 & 58.33 & 16.43 & 0.8 & 17 & .387 & -0.16 & 53.59 & 16.70 & 48.37 & 15.90 & 2.3 & 28 & .028 & 0.31 & -0.47 \\
\hline \multicolumn{18}{|l|}{ Maladjustment } \\
\hline Labour & 2.56 & 2.01 & 1.66 & 1.74 & 1.8 & 17 & .088 & 0.44 & 2.86 & 1.76 & 1.62 & 1.49 & 3.7 & 28 & .001 & 0.70 & -0.26 \\
\hline
\end{tabular}

Journal of Substance Abuse Treatment, 2017, 76, 28-35 


\begin{tabular}{|c|c|c|c|c|c|c|c|c|c|c|c|c|c|c|c|c|c|}
\hline Social & 2.72 & 1.87 & 1.72 & 1.52 & 2.3 & 17 & .032 & 0.53 & 3.28 & 1.55 & 1.89 & 1.69 & 3.6 & 28 & .001 & 0.89 & -0.36 \\
\hline Leisure & 2.50 & 1.75 & 1.50 & 1.20 & 2.4 & 17 & .024 & 0.57 & 3.48 & 1.50 & 2.17 & 1.60 & 4.1 & 28 & $<.001$ & 0.87 & -0.3 \\
\hline Partner & 1.72 & 1.70 & 1.33 & 1.60 & 0.7 & 17 & .493 & 0.22 & 4.17 & 1.25 & 2.10 & 1.71 & 5.5 & 28 & $<.001$ & 1.65 & -1.43 \\
\hline Family & 2.22 & 1.66 & 1.33 & 1.53 & 1.7 & 17 & .096 & 0.53 & 3.14 & 1.40 & 1.65 & 1.54 & 3.6 & 28 & .001 & 1.06 & -0.53 \\
\hline General & 2.94 & 1.55 & 1.88 & 1.77 & 3.0 & 17 & .007 & 0.68 & 3.90 & 1.20 & 2.17 & 1.65 & 4.3 & 28 & $<.001$ & 1.44 & -0.76 \\
\hline Total maladjustment & 14.67 & 8.55 & 9.44 & 7.69 & 2.3 & 17 & .031 & 0.61 & 20.83 & 6.73 & 11.62 & 8.20 & 5.1 & 28 & $<.001$ & 1.36 & -0.75 \\
\hline
\end{tabular}

$\mathrm{SD}$ = standard deviation; $\mathrm{df}=$ degree of freedom; $\mathrm{d}=$ Cohen 's $\mathrm{d}$

Journal of Substance Abuse Treatment, 2017, 76, 28-35

http://dx.doi.org/10.1016/j.jsat.2017.02.014 


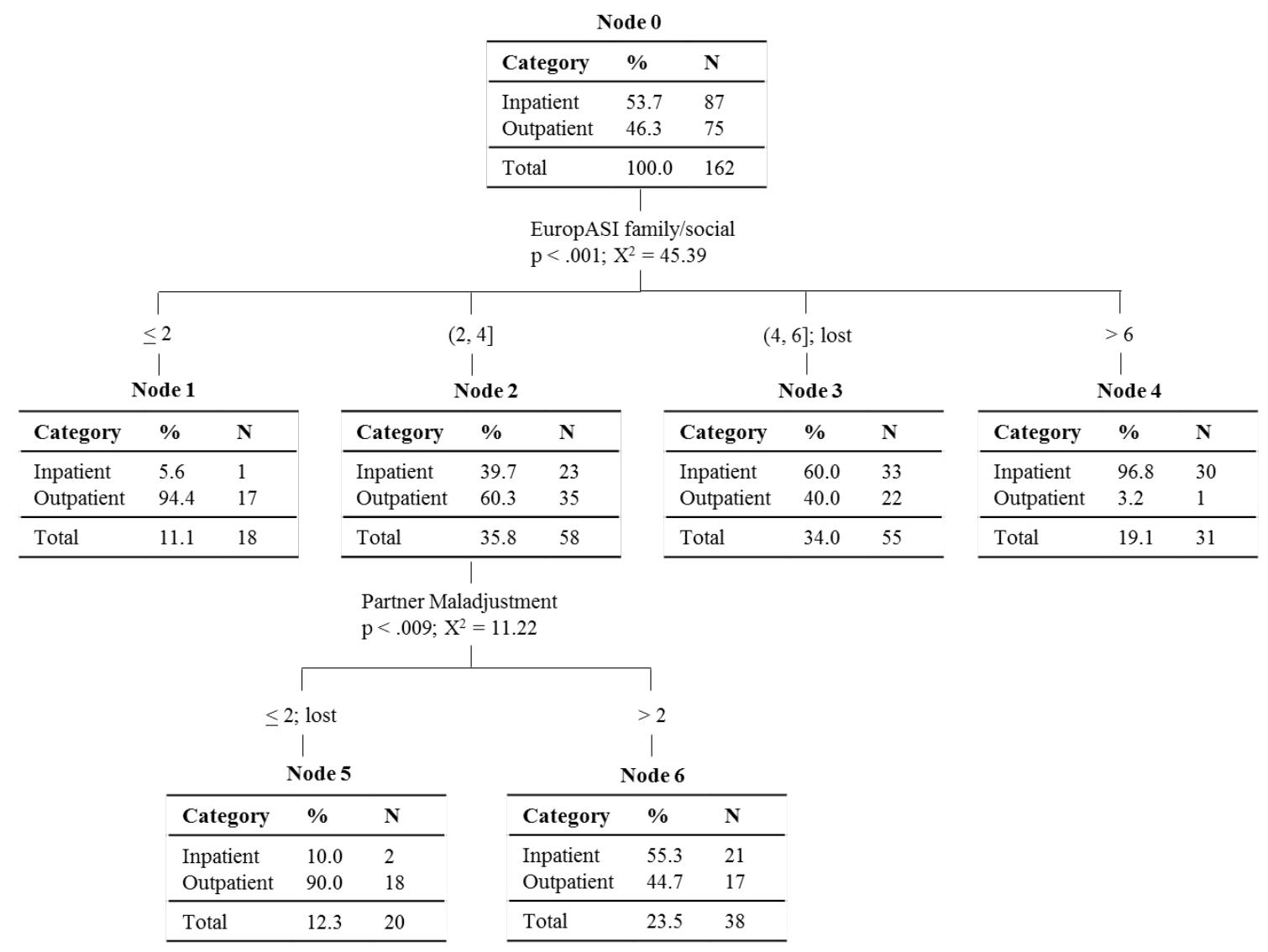

Figure 1 Results of CHAID analyses 